\title{
Spin-orbit-coupling-assisted roton softening and superstripes in a Rydberg-dressed Bose-Einstein Condensate
}

\author{
Hao Lyu and Yongping Zhang* \\ International Center of Quantum Artificial Intelligence for Science and Technology \\ (QuArtist) and Department of Physics, Shanghai University, Shanghai 200444, China
}

\begin{abstract}
Rotons can exist in ultracold atomic gases either with long-range interactions or with spin-orbitcoupled dispersions. We find that two different kinds of rotons coexist in a joint system combining long-range interactions and spin-orbit coupling. One roton originates from spin-orbit coupling and two others come from long-range interactions. Their softening can be controlled separately. The interesting new phenomenon which we find is that spin-orbit-coupled roton can push down the energy of one long-range-interaction roton. The spin-orbit coupling accelerates the softening of this roton. The post phase of spin-orbit-coupling-assisted roton softening and instability is identified as a superstripe.
\end{abstract}

\section{INTRODUCTION}

In quantum many-body systems, roton is a particular kind of collective excitations and features a parabolic-like dispersion relation at a finite momentum. Supersolid [1$5]$ is a unique phase of ground states and is characterized by the coexistence of crystalline densities due to spontaneous breaking of continuous translational symmetry and superfluidity associated with gauge symmetry breaking. These two different phenomena are connected by the socalled roton softening and instability. Varying relevant parameters gives rise to softening of the roton energy, and vanishing of the roton gap predicts that the homogeneous state hosting roton excitations is energetically unstable. Such a roton instability demands that the ground state possibly is a supersolid due to the simultaneous occupation of the original momentum and roton momentum where roton instability happens. Therefore, the roton softening and instability provides an accessible route to searching for supersolid phases [6, 7].

Recently, there is a big advance for the study of these twin phenomena in ultracold atomic gases, produced by their experimental implementations. In ultracold atoms, there are two different "arenas" to accommodate rotons and supersolids. One is with long-range interactions and the other is with special single-particle dispersion relations featuring multiple energy minima. Long-range interactions can form self-attractions around a certain momentum in momentum domain which generate a local minimum with infinite density of state, i.e., a roton [811]. Dominant long-range interactions can be experimentally realized with the assistance of optical cavities. Roton softening [12] and supersolid phases [13] have been observed in pioneering optical cavity experiments. Very recently, experimental achievements of Bose-Einstein condensation in strongly magnetic lanthanide atoms provide another promising perspective to measure the dipolar roton softening [7] and identify supersolids [14-17],

* yongping11@t.shu.edu.cn with the assistance of dominant long-range dipolar interactions. Furthermore, dipolar supersolids have been characterized by observations of their collective excitations from different aspects [18-22]. Rydberg-dressed Bose-Einstein condensates (BECs) offer another platform to realize long-range interactions [23-26]. They can be achieved by off-resonantly coupling BEC atoms to a Rydberg state [10, 23]. Unlike anisotropic dipolar interactions, nonlocal Rydberg-dressed interactions can be isotropic. Supersolid phases have been identified theoretically in Rydberg-dressed BECs [10, 27].

The other mechanism to introduce rotons and supersolids relates to single-particle dispersion relations. If single-particle dispersions possess multiple energy minima, the homogeneous ground state chooses to occupy one of them, another unoccupied minima are modified by repulsive atomic interactions to generate roton-like structures in collective excitations, no matter the interactions are short-range or long-range [28-30]. There already exists two experimental approaches to prepare such special single-particle dispersions. One utilizes periodically shaking optical lattices [31] and the other makes use of spin-orbit coupling induced by Raman coupling between lasers and atoms [32-36]. Roton softening has been examined in both shaking lattices and spin-orbit coupled gases [30, 31, 37]. Spin-orbit-coupled supersolids have also been observed experimentally in Ref. [38]. Because of their one-dimensional nature, they are called superstripes [39].

The combination of these two different arenas together endows rotons and supersolids with more interesting properties. Supersolids and superstripes have been investigated in spin-orbit-coupled systems with long-range interactions in different spatial dimensions [40-44]. Much attention has focused on supersolids. However, whether the rotons generated by the two different mechanisms can coexist and how the rotons in the combined arenas soften are not known yet.

In this paper, we address these questions by studying a spin-orbit-coupled BEC with long-range Rydbergdressed interactions. In atomic BECs, the pseudospin states of spin-orbit coupling could be hyperfine ground 
states. The coupling of hyperfine states by Raman lasers requires particular atoms [45]. Currently, all spin-orbitcoupled BEC experiments with hyperfine states are implemented in ${ }^{87} \mathrm{Rb}$ BECs $[32,37]$. While, the possible realization of Rydberg-dressed interactions does not need to choose specific atoms [23], which provides a hope to implement both $\mathrm{in}^{87} \mathrm{Rb}$ atoms. Until now, it is still a challenge to realize Rydberg-dressed BECs [46]. However, Rydberg-dressed BECs attract lots of current theoretical interest as an outstanding platform to explore phenomena of long-range interactions [47, 48].

We find that there exists two different kinds of rotons separately originating from single-particle spin-orbitcoupled dispersion and long-range interactions. The softening of these rotons can be adjusted independently. This tunability introduces the system into a new stage where the spin-orbit-coupled roton can assist the softening of long-range interaction-rotons. The consequence of such spin-orbit-coupling-assisted roton softening and instability is superstripe ground states. We characterize the superstripes by their collective excitation spectrum. Our study is organized as follows. In Sec. II, we present our theoretical frame for the analysis of roton and superstripe. In Sec. III, we uncover that two kinds of rotons with different origination mechanism can coexist, and spin-orbit-coupling-assisted roton softening will be demonstrated. The post-roton-softening phase, i.e., superstripes, will be discussed in Sec. IV, and the conclusion follows in Sec. V.

\section{MEAN-FIELD THEORY}

We start from a three dimensional atomic BEC and assume that harmonic traps along the transverse direction are strong enough so that the transverse motion of atoms is completely confined into the ground state of harmonic traps. After integrating over the transverse motion, we get a quasi-one-dimension (1D) spin-orbit-coupled BEC with Rydberg-dressed interactions. The mean-field energy functional of the system is,

$$
\begin{aligned}
E= & \int d x \psi^{\dagger}(x) H_{\mathrm{soc}} \psi(x) \\
& +\frac{1}{2} \sum_{i, j=1,2} \int d x g_{i j}\left|\psi_{i}(x)\right|^{2}\left|\psi_{j}(x)\right|^{2} \\
& +\frac{1}{2} \sum_{i, j=1,2} \int d x d x^{\prime} V_{i j}\left(x-x^{\prime}\right)\left|\psi_{i}(x)\right|^{2}\left|\psi_{j}\left(x^{\prime}\right)\right|^{2} .
\end{aligned}
$$

Here $\psi=\left(\psi_{1}, \psi_{2}\right)^{T}$ is the two-component wave function. $g_{i j}(i, j=1,2)$ characterize contact interactions between intra- and inter-species, which are proportional to $s$-wave scattering lengths and the atom number. $H_{\text {soc }}$ is the single-particle spin-orbit-coupled Hamiltonian,

$$
H_{\mathrm{soc}}=-\frac{1}{2} \frac{\partial^{2}}{\partial x^{2}}-i \gamma \frac{\partial}{\partial x} \sigma_{z}+\frac{\Omega}{2} \sigma_{x} .
$$

In experiments, the spin-orbit coupling term $-i \gamma \partial / \partial x \sigma_{z}$ can be artificially introduced into atoms by momentum exchanges between Raman lasers and atoms [30, 32, 37]. The spin-orbit coupling strength $\gamma=\hbar k_{\text {Ram }} / m$ relates to the wavelength $\lambda_{\text {Ram }}$ of Raman lasers with $k_{\text {Ram }}=$ $2 \pi / \lambda_{\text {Ram }}$, where $m$ is the atom mass. $\Omega$ is the Raman coupling and is proportional to the laser intensity, so that it can be easily tuned in experiments. In all our dimensionless equations, we choose the unit of momentum, length, and energy as $k_{\text {Ram }}, 1 / k_{\text {Ram }}$ and $\hbar^{2} k_{\text {Ram }}^{2} / m$, respectively. Therefore, under these units, the dimensionless parameter $\gamma=1$. However, the value of $\gamma$ can be varied by the periodic modulation of $\Omega$ according to the proposal in Ref. [49] and the experimental realization in [50]. The effective Rydberg-dressing potentials between intra- and inter-components are [10, 23, 51],

$$
V_{i j}(x)=\frac{\tilde{C}_{6}^{i j}}{x^{6}+R_{c}^{6}},
$$

with $\tilde{C}_{6}^{i j}$ being the interaction strengths of Rydbergdressing and $R_{c}$ being the blockade radius.

By minimizing the free energy $F=E-\mu N$ with $\mu$ being the chemical potential and $N$ being the total atom number, we get stationary Gross-Pitaevskii (GP) equations [5],

$$
\mu \psi=\left(H_{\mathrm{soc}}+H_{s}[\psi]+H_{\mathrm{Ryd}}[\psi]\right) \psi .
$$

Here $H_{s}$ denotes the contact interactions,

$$
H_{s}[\psi]=\left(\begin{array}{cc}
g_{11}\left|\psi_{1}\right|^{2}+g_{12}\left|\psi_{2}\right|^{2} & 0 \\
0 & g_{12}\left|\psi_{1}\right|^{2}+g_{22}\left|\psi_{2}\right|^{2}
\end{array}\right) .
$$

$H_{\text {Ryd }}$ represents the long-range Rydberg-dressed interactions,

$$
\begin{aligned}
& H_{\mathrm{Ryd}}[\psi]= \\
& \sum_{i=1,2} \int d x^{\prime}\left(\begin{array}{cc}
V_{1 i}\left(x^{\prime}-x\right)\left|\psi_{i}\left(x^{\prime}\right)\right|^{2} & 0 \\
0 & V_{2 i}\left(x^{\prime}-x\right)\left|\psi_{i}\left(x^{\prime}\right)\right|^{2}
\end{array}\right) .
\end{aligned}
$$

Once we know the ground-state wave function $\psi$ and corresponding $\mu$, we can study their collective excitations by adding perturbations into the ground state. Therefore total wave functions are,

$$
\Psi_{1,2}=e^{-i \mu t}\left[\psi_{1,2}(x)+u_{1,2}(x) e^{-i \omega t}+v_{1,2}^{*}(x) e^{i \omega t}\right],
$$

where $\omega$ is the excitation energy, and $u_{1,2}(x), v_{1,2}(x)$ are perturbation amplitudes, satisfying the normalization condition, $\sum_{l=1,2} \int d x\left(\left|u_{l}(x)\right|^{2}-\left|v_{l}(x)\right|^{2}\right)=1$. After substituting $\Psi_{1,2}$ into the time-dependent version of Eq. (4) and keeping linear terms of $u$ and $v$, we get Bogoliubov-de Gennes (BdG) equations [52],

$$
\mathcal{L}_{1} \phi+\mathcal{L}_{2}[\phi]=\omega \phi,
$$

with $\phi=\left(u_{1}(x), u_{2}(x), v_{1}(x), v_{2}(x)\right)^{T}$. The matrix $\mathcal{L}_{1}$ is

$$
\mathcal{L}_{1}=\left(\begin{array}{cc}
H_{\mathrm{soc}}+A-\mu & B \\
B^{*} & -H_{\mathrm{soc}}-A^{*}-\mu
\end{array}\right),
$$


with

$$
A=H_{s}[\psi]+H_{\mathrm{Ryd}}[\psi]+\left(\begin{array}{cc}
g_{11}\left|\psi_{1}\right|^{2} & g_{12} \psi_{1}^{*} \psi_{2} \\
g_{12} \psi_{1} \psi_{2}^{*} & g_{22}\left|\psi_{2}\right|^{2}
\end{array}\right)
$$

and

$$
B=\left(\begin{array}{cc}
g_{11} \psi_{1}^{2} & g_{12} \psi_{1} \psi_{2} \\
g_{12} \psi_{1} \psi_{2} & g_{22} \psi_{2}^{2}
\end{array}\right)
$$

The matrix $\mathcal{L}_{2}[\phi]$ is given by

$$
\mathcal{L}_{2}[\phi]=\int d x^{\prime}\left(\begin{array}{ll}
M_{1}\left(x, x^{\prime}\right) & M_{2}\left(x, x^{\prime}\right) \\
M_{2}^{*}\left(x, x^{\prime}\right) & M_{1}^{*}\left(x, x^{\prime}\right)
\end{array}\right) \phi\left(x^{\prime}\right)
$$

with

$$
\begin{aligned}
& M_{1}\left(x^{\prime}, x\right)= \\
& \left(\begin{array}{ll}
V_{11}\left(x^{\prime}-x\right) \psi_{1}^{*}\left(x^{\prime}\right) \psi_{1}(x) & V_{12}\left(x^{\prime}-x\right) \psi_{2}^{*}\left(x^{\prime}\right) \psi_{1}(x) \\
V_{12}\left(x^{\prime}-x\right) \psi_{1}^{*}\left(x^{\prime}\right) \psi_{2}(x) & V_{22}\left(x^{\prime}-x\right) \psi_{2}^{*}\left(x^{\prime}\right) \psi_{2}(x)
\end{array}\right), \\
& M_{2}\left(x^{\prime}, x\right)= \\
& \left(\begin{array}{ll}
V_{11}\left(x^{\prime}-x\right) \psi_{1}\left(x^{\prime}\right) \psi_{1}(x) & V_{12}\left(x^{\prime}-x\right) \psi_{2}\left(x^{\prime}\right) \psi_{1}(x) \\
V_{12}\left(x^{\prime}-x\right) \psi_{1}\left(x^{\prime}\right) \psi_{2}(x) & V_{22}\left(x^{\prime}-x\right) \psi_{2}\left(x^{\prime}\right) \psi_{2}(x)
\end{array}\right) .
\end{aligned}
$$

In the following, we solve GP equations (4) to search for ground states and solve BdG equations (6) to analyze the collective excitation spectrum of corresponding ground states. We set $g_{11}=g_{22}=g_{12}=g$, since they are approximately the same in experiments [32]. The two-component Rydberg-dressed BEC may be realized by coupling two ground hyperfine states to different Rydberg states $[44,51]$. The strength of long-range interactions $\tilde{C}_{6}^{i j}$ and the blockade radius $R_{c}$ depend on the two-photon detuning and Rabi frequency of excitation lasers, which are tunable in experiments [23]. In this work, we choose $V_{11}(x)=V_{22}(x)=V_{12}(x)=V(x)$ for simplicity, so that $\tilde{C}_{6}^{i j}=\tilde{C}_{6}$. For further convenience, we transform $V(x)$ into the momentum space, i.e., $\tilde{V}(k)=$ $\int d x V(x) \exp (i k x)=\tilde{V}_{0} f(k)$, with $\tilde{V}_{0}=2 \pi \tilde{C}_{6} / 3 R_{c}^{5}$ and $f(k)=\frac{1}{2} e^{-|k| R c / 2}\left[e^{-|k| R_{c} / 2}+\cos \left(\sqrt{3}|k| R_{c} / 2\right) \quad+\right.$ $\left.\sqrt{3} \sin \left(\sqrt{3}|k| R_{c} / 2\right)\right]$. We use parameters $\tilde{V}_{0}$ and $R_{c}$ to characterize the Rydberg-dressed interactions. The validity of theoretical mean-field frame in our quasi-1D system is examined by the calculation of quantum depletion demonstrated the appendix.

\section{SPIN-ORBIT-COUPLING-ASSISTED ROTON SOFTENING}

Without the spin-orbit coupling $(\gamma=0, \Omega=0)$, it is known that the collective excitation spectrum $\omega(q)$ of a BEC with long-range interactions can be analytically calculated from the BdG equations [53]. In collective excitations, there are two branches [54, 55]; one is spindensity excitations $\omega=q^{2} / 2$, and the other is density
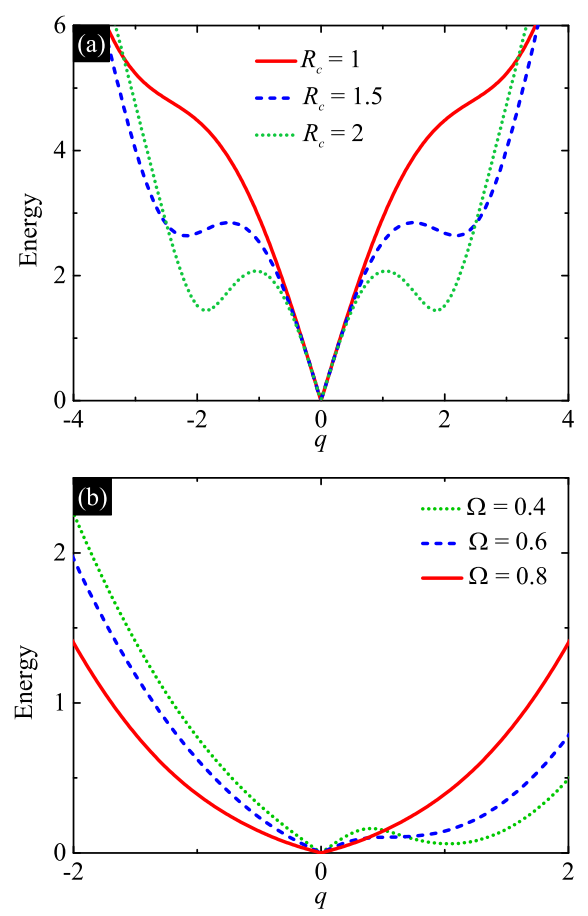

FIG. 1. (a) The density excitation of a Rydberg-dressed BEC for various $R_{c}$ with a fixed $\tilde{V}_{0}=10$. (b) The lower branch of the collective excitation of the plane-wave ground state in a spin-orbit coupled BEC without long-range interactions. The spin-orbit coupling strength is set as $\gamma=0.6$. In both (a) and (b), $s$-wave interaction strength is fixed as $g=0.5$.

excitations,

$$
\omega=\frac{1}{2} \sqrt{q^{4}+4 q^{2}[g+\tilde{V}(q)]}
$$

The density branch is consistent with the collective excitation spectrum of a single component with long-range interactions [10]. In Fig. 1(a), the density branch as a function of $R_{c}$ is shown for a fixed $\tilde{V}_{0}$. We check that $\tilde{V}(q)$ becomes attractive around $|q| \sim 4.3 / R_{c}$. It predicts [6] that the roton momentum $q_{\text {rot }}$ where minimum of roton energy happens can be characterized by the blockade radius, which is consistent with the finding in Ref. [10]. One of the interesting features of Rydberg-dressed rotons is that rotons appear in a pair and symmetrically distribute at $q_{\text {rot }} \sim \pm 4.3 / R_{c}$. Increasing $R_{c}$ leads to the shrinking of roton minimum and meanwhile the reduction of roton gap. Thus, roton softening can be introduced by increasing $R_{c}$ [see Fig. 1(a)]. Once the roton gap is closed, roton instability happens and further increasing $R_{c}$ leads to the roton energy becoming complex-valued.

As mentioned above, we unravel the mechanism of the existence of roton softening in a Rydberg-dressed BEC. In the following, we show that roton softening can exist in a spin-orbit-coupled BEC. The dispersion relation of the single-particle spin-orbit-coupled Hamiltonian $H_{\mathrm{soc}}$ possesses two bands and the lowest band has two degenerate energy minima. These two minima give rise to the 
roton spectrum. In order to show this, we assume that the ground state is a plane-wave, so that the wave functions are $\psi(x)=e^{i k x}\left(\varphi_{1}, \varphi_{2}\right)^{T}[28,56]$ with $k$ being the ground-state quasimomentum. $\varphi_{1,2}$ are independent of spatial coordinates and satisfy the renormalization condition $\left|\varphi_{1}\right|^{2}+\left|\varphi_{2}\right|^{2}=1$. With this ansatz, the energy functional becomes

$$
\begin{aligned}
E= & \frac{1}{2} k^{2}+\gamma k\left(\left|\varphi_{1}\right|^{2}-\left|\varphi_{2}\right|^{2}\right)+\frac{\Omega}{2}\left(\varphi_{1}^{*} \varphi_{2}+\varphi_{1} \varphi_{2}^{*}\right) \\
& +\frac{1}{2}\left(g+\tilde{V}_{0}\right) .
\end{aligned}
$$

From the above expression, it is clear that long-range and contact interactions contribute only an overall shift of the energy since all their coefficients are the same. This leads to the fact that the properties of the ground state should be similar to that of the single-particle case. For $\Omega<2 \gamma^{2}$, minimization of the energy functional results in two energy minima laying at quasimomentum $k_{ \pm}= \pm \gamma \sqrt{1-\Omega^{2} / 4 \gamma^{2}}$, and the plane-wave ground state spontaneously chooses one of them to occupy. By using the results of the minimization with the calculated chemical potential and assuming $u_{1,2}(x)=u_{1,2} e^{i(k+q) x}$ and $v_{1,2}(x)=v_{1,2} e^{-i(k-q) x}$ [28] with $q$ being the perturbation quasimomentum, we calculate the $\mathrm{BdG}$ equations to obtain the collective excitation spectrum $\omega(q)$ of the plane-wave ground state. Figure 1(b) demonstrates roton softening by varying the Raman coupling $\Omega$ without longrange interactions $\left(\tilde{V}_{0}=0\right)$. The spin-orbit-coupled roton only exists in one side of $q$. This is because the planewave ground state that we use to plot Fig. 1(b) spontaneously occupies $k_{-}$. Since the contact interactions $g$ contributes positive energy to the system, they displace single-particle dispersion upwards and meanwhile shape the dispersion around $k_{-}$(corresponding to $q=0$ in the perturbation momentum frame) into a linear form. A phonon mode can be formed in this mechanism. Therefore, the other unoccupied single-particle minimum at $k_{+}$ appears as a roton-like structure in collective excitations. In the perturbation quasimomentum frame, a roton sits at $q_{\text {rot }} \approx k_{+}-k_{-}=2 \gamma \sqrt{1-\Omega^{2} / 4 \gamma^{2}}$. Decrease of $\Omega$ causes roton softening, as well as the increase of roton momentum [see Fig. 1(b)]. Further decrease of $\Omega$ can not result in roton instability. This is because there is no phase transition from the plane-wave ground state to superstripes, with all equal interaction coefficients [56]. If we make the interaction coefficients unequal, roton instability occurs after the softening and it features negative roton energy [30].

We conclude that the momentum of Rydberg-dressed roton is governed by the blockade radius $R_{c}$, while the spin-orbit-coupled-roton's momentum relates to spinorbit coupling parameters $\gamma$ and $\Omega$. They have completely different originations, which intuitively makes their coexistence possible. We perform numerical calculations to confirm this expectation. The collective excitation of a BEC in the presence of both spin-orbit coupling and long-range Rydberg-dressed interactions
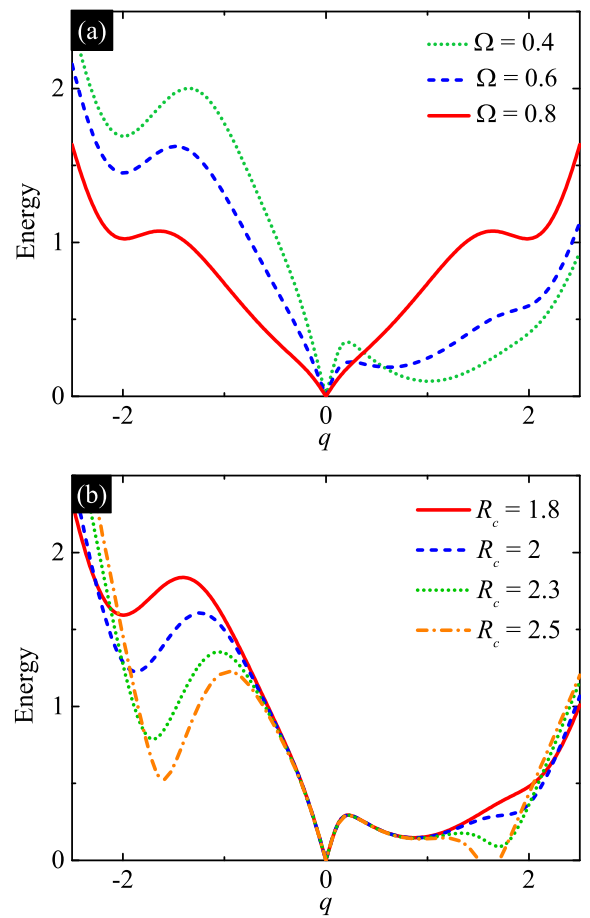

FIG. 2. Collective excitation spectrum of the Rydbergdressed BEC with spin-orbit coupling, in which we choose $\gamma=0.6, g=0.5$, and $\tilde{V}_{0}=10$. (a) Evolution of the spectrum for various $\Omega$ with a fixed $R_{c}=1.8$. (b) Evolution of the spectrum for various $R_{c}$ with a fixed $\Omega=0.5$. For the line with $R_{c}=2.5$, the right-hand Rydberg-dressed roton become unstable with complex-valued energy which is not shown in the plot.

are demonstrated in Fig. 2(a). For $R_{c}=1.8$, we see that there are only Rydberg-dressed rotons sitting at $q_{\text {rot }} \sim \pm 4.3 / R_{c} \sim \pm 2.2$ symmetrically when $\Omega$ is large [see the line with $\Omega=0.8$ in Fig. 2(a)]. This is expected since for a larger $\Omega$ there is no spin-orbit-coupled roton even without long-range interactions [see the line with $\Omega=0.8$ in Fig. 1(b)]. Decreasing $\Omega$ leads to the appearance of the spin-orbit-coupled roton sitting approximately at $2 \gamma \sqrt{1-\Omega^{2} / 4 \gamma^{2}}$. The newborn roton pushes down the spectrum at right-hand side, and pushes up the left-hand side spectrum. However, it does not affect the location of Rydberg-dressed rotons [see the lines with $\Omega=0.6$ and 0.4 in Fig. 2(a)]. From the line with $\Omega=0.4$ in Fig. 2(a), where the spin-orbit-coupled roton dominates, the Rydberg-dressed roton at right side fades away while the left side roton survives.

Consequently, the two different kinds of rotons can exist independently. Meanwhile, the spin-orbit-coupled roton dramatically pushes spectrum on its side down, which provides a new phenomenon for the softening of the Rydberg-dressed roton. In Fig. 2(b) we show the Rydberg-dressed roton softening by varying $R_{c}$. We start from the coexistence case with $R_{c}=1.8$ (the red line). Due to the spin-orbit-coupled roton, the spectrum is completely asymmetric with respect to $q=0$. The right-hand 

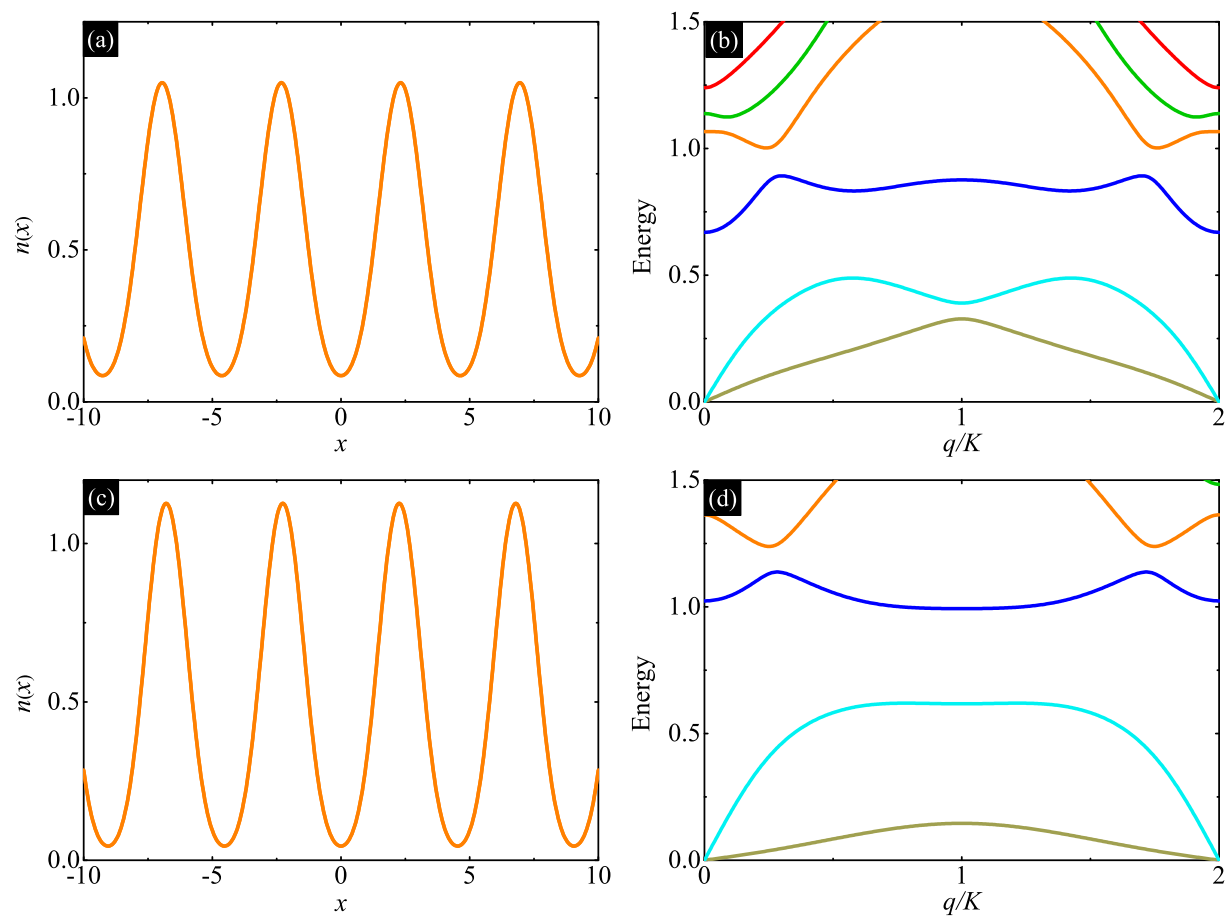

FIG. 3. Density profiles (a,c) and excitation spectrums (b,d) of the superstripe phase. We choose $\Omega=0.4$ in (a,b) and 0.8 in (c,d). The other parameters are $\gamma=0.6, g=0.5, \tilde{V}_{0}=10$, and $R_{c}=3$.

side Rydberg-dressed roton is obviously lower than the left-hand one. Surprisingly, the increase of $R_{c}$ quickly brings the right-hand roton into the unstable situation, while the left-hand one softens but still has a large roton gap [see the line with $R_{c}=2.5$ in Fig. 2(b)]. Very interestingly, the change of $R_{c}$ does not affect the spinorbit-coupled roton.

Therefore, we can have parameter regimes in which only one Rydberg-dressed roton becomes unstable. This is fully distinguishable with the case without spin-orbit coupling where paired Rydberg-dressed rotons soften and are unstable in the same time. The reason of one unstable roton is that it is the spin-orbit-coupled roton that helps to lower its energy to prepare for the occurring of instability. Thus, the spin-orbit coupling plays a role of an assistance for one Rydberg-dressed roton softening and instability. It can enhance the roton softening and accelerate instability to happen. We emphasize that for our parameters the spin-orbit-coupled roton can not become unstable and the instability always happens in the Rydberg-dressed rotons.

\section{POST-ROTON-INSTABILITY PHASE: SUPERSTRIPE}

Now we are in the position to ask: what are properties of post-roton-instability phase induced by such a new mechanism of the spin-orbit-coupling-assisted roton softening. To address this question, we numerically find the existence of superstripe ground state. Considering the periodic density distributions of the superstripe, we assume that the wave functions are periodic and can be expressed in a plane-wave basis [39, 57],

$$
\psi(x)=\sum_{n=-L}^{L}\left(\begin{array}{l}
\psi_{1}^{(n)} \\
\psi_{2}^{(n)}
\end{array}\right) e^{i n K x},
$$

where $\psi_{1}^{(n)}$ and $\psi_{n}^{(n)}$ satisfy the renormalization condition $\sum_{n}\left(\left|\psi_{1}^{(n)}\right|^{2}+\left|\psi_{2}^{(n)}\right|^{2}\right)=1$, and $L$ is the cutoff of the plane-wave modes. This ansatz indicates that the period of wave functions is relevant to $K$, whose value shall be determined by minimizing corresponding energy functional obtained by substituting Eq. (10) into Eq. (1). Minimization procedure demonstrates that only the coefficients of odd-numbered plane-wave modes (i.e., $e^{ \pm i K x}, e^{ \pm i 3 K x}$, etc.) are nonzero. Therefore, the period of wave functions is numerically exact $2 \pi / K$ and the density period $\pi / K$. These results are similar to that in spin-orbit-coupled BECs without Rydberg-dressed interactions $[39,57]$. Two typical density distributions are shown in Fig. 3(a,c) for $\Omega=0.4$ and 0.8 respectively. We see that $\Omega$ has little impact on density periodicity. The density period $\pi / K$ is $\sim 4.6$, which is consistent with the predicted value from the roton instability. For $R_{c}=3$ which is used in Fig. 3, we check the roton instability centered around $q_{\text {rot }} \sim 1.36$, which predicts that the period of post-roton-instability phase should be $2 \pi / q_{\text {rot }} \sim 4$.6. The density period for various $\Omega$ is demonstrated in Fig. 4(a). It confirms that density period is 

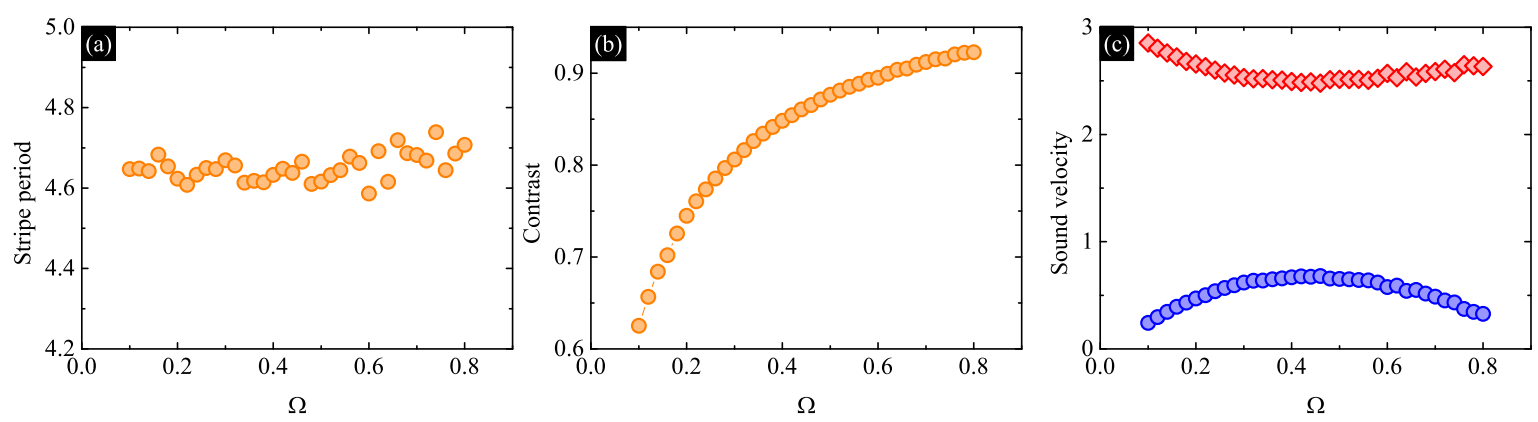

FIG. 4. The period (a) and contrast (b) of the superstripes as a function of the Raman coupling strength $\Omega$. (c) Sound velocity of the upper (circles) and lower (squares) phonon branches as a function of the Raman coupling strength $\Omega$. The parameters are $\gamma=0.6, g=0.5, R_{c}=3$, and $\tilde{V}_{0}=10$.

insensitive to $\Omega$.

We further characterize the superstripe ground states by their collective excitation spectrum. It is calculated by substituting the superstripe wave functions into BdG equations (6) and using the following perturbation amplitudes,

$$
\begin{aligned}
& u_{1,2}(x)=e^{i q x} \sum_{n=-L}^{L} u_{1,2}^{(n)} e^{i(2 n+1) K x} \\
& v_{1,2}(x)=e^{i q x} \sum_{n=-L}^{L} v_{1,2}^{(n)} e^{i(2 n+1) K x} .
\end{aligned}
$$

The reason for the choice of above amplitudes is that the BdG equations are periodic with the period being $\pi / K$, as a result of the periodic density of the superstripes. Therefore, the amplitudes should be Bloch states with $q$ being perturbation quasimomentum and we expand their periodic parts by a plane-wave basis. The results obtained by diagonalizing the BdG equations are shown in Fig. 3(b,d), corresponding to the superstripes plotted in Fig. 3(a,c) respectively. There is a slight difference between the two superstripes in Fig. 3(a,c). But the collective excitation spectrum are obviously different, which features a Bloch band-gap structure. The gap sizes in Fig. 3(d) is clearly larger than these in Fig. 3(b). This is because the density of superstripe in Fig. 3(c) have a higher amplitude than that in Fig. 3(a). A high density will open a large gap in collective excitations. We label the density amplitude by the contrast of superstripe, which is defined as

$$
C=\frac{n_{\max }-n_{\min }}{n_{\max }+n_{\min }}
$$

with $n_{\max }$ and $n_{\min }$ being the maximum and minimum of density respectively. The contrast as a function of $\Omega$ is plotted in Fig. 4(b). It increases with the increase of the $\Omega$. Thus, we expect that a larger Raman coupling gives rise to larger gap sizes in collective excitations.

In the long wavelength regime, the lowest two bands of collective excitation spectrum are phonon modes with different sound velocities. The existence of these two gapless Goldstone modes $[39,58]$ is guaranteed by two spontaneous symmetry breaking of superstripes, one of which is continuous translational symmetry breaking and the other is gauge symmetry breaking. Sound velocities relate to slopes of phonon modes are analyzed as a function of $\Omega$, and the result is shown in Fig. 4(c). The velocity of the upper phonon branch decreases slowly with increasing $\Omega$ [see circles in Fig 4(c)]. However, the velocity of the lower phonon branch slowly increases and then decreases as a function of $\Omega$ [see squares in Fig 4(c)]. Sound velocity shows a jump around phase transition from plane-wave to superstripe phases. Therefore, the phase transition is first order.

Finally, we emphasize that the superstripes studied above are ground states supported by spin-orbitcoupling-assisted softening and instability. Without spinorbit coupling, the ground states of relevant parameters are density homogeneous with roton excitations.

\section{CONCLUSION}

Long-range Rydberg-dressed interactions and spinorbit coupling can separately generate collective excitations with roton structures. In a system with both Rydberg-dressed interactions and spin-orbit coupling, two different kinds of rotons from different originations can coexist. The location and softening of these rotons are adjustable independently. The interplay of them leads to an interesting phenomenon that the spin-orbitcoupled roton can assist Rydberg-dressed roton softening. The post-roton-instability phase is a superstripe, which is identified by analyzing their collective excitations. We conclude that spin-orbit coupling provides a possible means to accelerate roton softening.

\section{ACKNOWLEDGEMENT}

We thank Zhaoxin Liang and Zhu Chen for the useful help. This work is supported by the NSF of China (Grant 

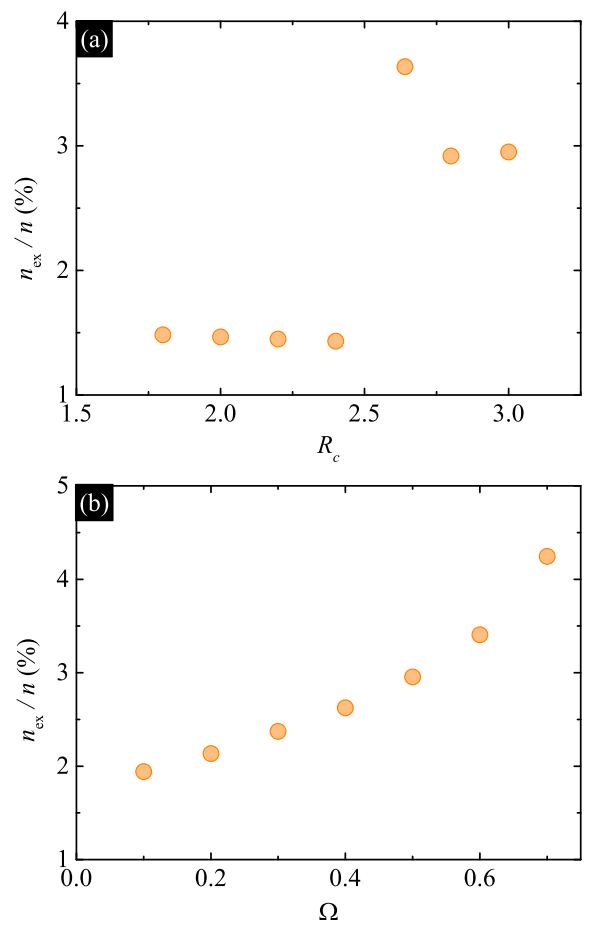

FIG. 5. Quantum depletion $n_{\mathrm{ex}} / n$ of the Rydberg-dressed BEC with spin-orbit coupling. (a) Quantum depletion as a function of $R_{c}$ with a fixed $\Omega=0.5$. (b) Quantum depletion as a function of $\Omega$ with a fixed $R_{c}=3$. Other parameters are $\gamma=0.6, g=0.5, \Omega=0.5, \tilde{V}_{0}=10$, and $n=200 k_{\text {Ram }}$. A cutoff $q_{c}=0.01 k_{\text {Ram }}$ is introduced to prevent divergence.

Nos. 1174219 and 11974235), the Thousand Young Talents Program of China, and the Eastern Scholar and Shuguang (Program No. 17SG39) Program. H. L. acknowledges the support by China Postdoctoral Science Foundation (Grant No. 2019M661457).

\section{Appendix A: Quantum depletion}

In atomic BECs, quantum fluctuation induces a fraction of the condensate depleted even at zero temperature. The mean-field theory is valid when the number of depleted atoms is much smaller than the total atom number. Quantum depletion has been calculated in spinorbit-coupled BECs [57, 59-62]. In the following, we show that quantum depletion of our system is small and the mean-field description is reasonable.

We start from three-dimensional GP equations and consider the atoms are strongly confined in the transverse direction. The transverse wave functions can be assumed to have a Gaussian shape. By integrating the transverse motion, we can obtain the quasi-1D GP equations. At zero temperature, the density of depleted atoms can be calculated as [57]

$$
n_{\mathrm{ex}}=\sum_{j} \sum_{l=1,2} \sum_{q \neq 0} \int d x\left|v_{q, l}^{(j)}(x)\right|^{2} .
$$

Here, the superscript $j$ labels the $j$ th Bogoliubov band. The perturbation amplitude $v_{q, l}^{j}(x)$ depends on the quasimomentum $q$, which is given in the main text. Here, the transverse excitations are neglected due to the strong confinement. In Fig. 5(a), we show that by tuning the Rydberg blockade radius, the quantum depletion $n_{\text {ex }} / n$ jumps around the phase transition from planewave $\left(R_{c}<2.5\right)$ to superstripe $\left(R_{c}>2.5\right)$ phases. $n$ is the BEC density, here we use a value $n=200 k_{\text {Ram }}$ that is typical in usual BEC experiments. In the calculation, a cutoff of $q$ must be introduced to avoid divergence at very close to $q=0$ [63], here the cutoff we used is $q_{c}=0.01 k_{\text {Ram }}$. For the superstripe phase, the quantum depletion increase with the increase of the Rabi frequency, as shown in Fig. 5(b). In our parameter regimes, $n_{\mathrm{ex}} / n$ is less than $5 \%$, which indicates the validity of our mean-field theory.
[1] O. Penrose and L. Onsager, Bose-Einstein condensation and liquid Helium, Phys. Rev. 104, 576 (1956).

[2] E. P. Gross, Unified theory of interacting Bosons, Phys. Rev. 106, 161 (1957).

[3] G. Chester, Speculations on Bose-Einstein condensation and quantum crystals, Phys. Rev. A 2, 256 (1970).

[4] A. J. Leggett, Can a solid be "Superfluid"? Phys. Rev. Lett. 25, 1543 (1970).

[5] Y. Pomeau and S. Rica, Dynamics of a model of supersolid, Phys. Rev. Lett. 72, 2426 (1994).

[6] F. Ancilotto, M. Rossi, and F. Toigo, Supersolid structure and excitation spectrum of soft-core bosons in three dimensions, Phys. Rev. A 88, 033618 (2013).

[7] D. Petter, G. Natale, R. M. W. van Bijnen, A. Patscheider, M. J. Mark, L. Chomaz, and F. Ferlaino, Probing the roton excitation spectrum of a stable dipolar Bose gas, Phys. Rev. Lett. 122, 183401 (2019).
[8] L. Santos, G. V. Shlyapnikov, and M. Lewenstein, Roton-Maxon spectrum and stability of trapped dipolar Bose-Einstein Condensates, Phys. Rev. Lett. 90, 250403 (2003).

[9] D. H. J. O'Dell, S. Giovanazzi, and G. Kurizki, Rotons in gaseous Bose-Einstein condensates irradiated by a laser, Phys. Rev. Lett. 90, 110402 (2003).

[10] N. Henkel, R. Nath, and T. Pohl, Three-dimensional Roton excitations and supersolid formation in Rydberg-excited Bose-Einstein Condensates, Phys. Rev. Lett. 104, 195302 (2010).

[11] Y. Kora and M. Boninsegni, Patterned supersolids in dipolar Bose systems, J. Low Temp. Phys. 197, 337 (2019).

[12] R. Mottl, F. Brennecke, K. Baumann, R. Landig, T. Donner, and T. Esslinger, Roton-type mode softening in a quantum gas with cavity-mediated long-range interac- 
tions, Science 336, 1570 (2012).

[13] J. Léonard, A. Morales, P. Zupancic, T. Esslinger, and T. Donner, Supersolid formation in a quantum gas breaking a continuous translational symmetry, Nature (London) 543, 87 (2017).

[14] L. Chomaz, R. M. W. van Bijnen, D. Petter, G. Faraoni, S. Baier, J. H. Becher, M. J. Mark, F. Wächtler, L. Santos, and F. Ferlaino, Observation of roton mode population in a dipolar quantum gas, Nat. Phys. 14, 442 (2018).

[15] F. Böttcher, J.-N. Schmidt, M. Wenzel, J. Hertkorn, M. Guo, T. Langen, and T. Pfau, Transient supersolid properties in an array of dipolar quantum droplets, Phys. Rev. X 9, 011051 (2019).

[16] L. Chomaz, D. Petter, P. Ilzhöfer, G. Natale, A. Trautmann, C. Politi, G. Durastante, R. M. W. van Bijnen, A. Patscheider, M. Sohmen, M. J. Mark, and F. Ferlaino, Long-lived and transient supersolid behaviors in dipolar quantum gases, Phys. Rev. X 9, 021012 (2019).

[17] L. Tanzi, E. Lucioni, F. Famá, J. Catani, A. Fioretti, C. Gabbanini, R. N. Bisset, L. Santos, and G. Modugno, Observation of a dipolar quantum gas with metastable supersolid properties, Phys. Rev. Lett. 122, 130405 (2019).

[18] J. Léonard, A. Morales, P. Zupancic, T. Donner, and T. Esslinger, Monitoring and manipulating Higgs and Goldstone modes in a supersolid quantum gas, Science 358, 1415 (2017).

[19] G. Natale, R. M. W. van Bijnen, A. Patscheider, D. Petter, M. J. Mark, L. Chomaz, and F. Ferlaino, Excitation spectrum of a trapped dipolar supersolid and its experimental evidence, Phys. Rev. Lett. 123, 050402 (2019).

[20] L. Tanzi, S. M. Roccuzzo, E. Lucioni, F. Famà, A. Fioretti, C. Gabbanini, G. Modugno, A. Recati, and S. Stringari, Supersolid symmetry breaking from compressional oscillations in a dipolar quantum gas, Nature (London) 574, 382 (2019).

[21] M. Guo, F. Böttcher, J. Hertkorn, J.-N. Schmidt, M. Wenzel, H. P. Büchler, T. Langen, and T. Pfau, The low-energy Goldstone mode in a trapped dipolar supersolid, Nature (London) 574, 386 (2019).

[22] J. Hertkorn, F. Böttcher, M. Guo, J.-N. Schmidt, T. Langen, H. P. Büchler, and T. Pfau, Fate of the amplitude mode in a trapped dipolar supersolid, Phys. Rev. Lett. 123, 193002 (2019).

[23] M. Saffman, T. G. Walker, and K. Mölmer, Quantum information with Rydberg atoms, Rev. Mod. Phys. 82, 2313 (2010).

[24] P. Schauß, J. Zeiher, T. Fukuhara, S. Hild, M. Cheneau, T. Macrì, T. Pohl, I. Bloch, and C. Gross, Crystallization in Ising quantum magnet, Science 347, 1455 (2015).

[25] O. Firstenberg, C. S. Adams, and S. Hofferberth, Nonlinear quantum optics mediated by Rydberg interactions, J. Phys. B: At. Mol. Opt. Phys. 49, 152003 (2016).

[26] A. Browaeys and T. Lahaye, Many-body physics with individually controlled Rydberg atoms, Nat. Phys. 16, $132(2020)$.

[27] N. Henkel, F. Cinti, P. Jain, G. Pupillo, and T. Pohl, Supersolid vortex crystals in Rydberg-dressed BoseEinstein condensates, Phys. Rev. Lett. 108, 265301 (2012).

[28] G. I. Martone, Y. Li, L. P. Pitaevskii, and S. Stringari, Anisotropic dynamics of a spin-orbit-coupled BoseEinstein condensate, Phys. Rev. A 86, 063621 (2012).

[29] W. Zheng, Z.-Q. Yu, X. Cui, and H. Zhai, Properties of
Bose gases with the Raman-induced spin-orbit coupling, J. Phys. B: At. Mol. Opt. Phys. 46, 134007 (2013).

[30] M. A. Khamehchi, Y. Zhang, C. Hamner, T. Busch, and P. Engels, Measurement of collective excitations in a spin-orbit-coupled Bose-Einstein condensate, Phys. Rev. A 90, 063624 (2014).

[31] L.-C. Ha, L. W. Clark, C. V. Parker, B. M. Anderson, and C. Chin, Roton-maxon excitation spectrum of Bose condensates in a shaken optical lattice, Phys. Rev. Lett. 114, 055301 (2015).

[32] Y.-J. Lin, K. Jiménez-García, and I. B. Spielman, Spinorbit-coupled Bose-Einstein condensates, Nature (London) 471, 83 (2011).

[33] V. Galitski and I. B. Spielman, Spin-orbit coupling in quantum gases, Nature (London) 494, 49 (2013).

[34] N. Goldman, G. Juzeliūnas, P. Öhberg, and I. B. Spielman, Light-induced gauge fields for ultracold atoms, Rep. Prog. Phys. 77, 126401 (2014).

[35] H. Zhai, Degenerate quantum gases with spin-orbit coupling: a review, Rep. Prog. Phys. 78, 026001 (2015).

[36] Y. Zhang, M. E. Mossman, T. Busch, P. Engels, and C. Zhang, Properties of spin-orbit-coupled Bose-Einstein condensates, Front. Phys. 11, 118103 (2016).

[37] S.-C. Ji, L. Zhang, X.-T. Xu, Z. Wu, Y. Deng, S. Chen, and J.-W. Pan, Softening of roton and phonon modes in a Bose-Einstein condensate with spin-orbit coupling, Phys. Rev. Lett. 114, 105301 (2015).

[38] J. -R. Li, J. Lee, W. Huang, S. Burchesky, B. Shteynas, F. Ç. Top, A. O. Jamison, and W. Ketterle, A stripe phase with supersolid properties in spin-orbit-coupled Bose-Einstein condensates, Nature (London) 543, 91 (2017).

[39] Y. Li, G. I. Martone, L. P. Pitaevskii, and S. Stringari, Superstripes and the excitation spectrum of a spin-orbitcoupled Bose-Einstein condensate, Phys. Rev. Lett. 110, 235302 (2013).

[40] Y. Deng, J. Cheng, H. Jing, C.-P. Sun, and S. Yi, Spin-orbit-coupled dipolar Bose-Einstein condensates, Phys. Rev. Lett. 108, 125301 (2012).

[41] R. M. Wilson, B. M. Anderson, and C. W. Clark, Meron ground state of Rashba spin-orbit-coupled dipolar Boson, Phys. Rev. Lett. 111, 185303 (2013).

[42] S. Gopalakrishnan, I. Martin, and E. A. Demler, Quantum quasicrystals of spin-orbit-coupled dipolar Bosons, Phys. Rev. Lett. 111, 185304 (2013).

[43] H. Lü, S.-B. Zhu, J. Qian, and Y.-Z. Wang, Spinorbit coupled Bose-Einstein condensates with Rydbergdressing interactions, Chin. Phys. B 24, 090308 (2015).

[44] W. Han, X.-F. Zhang, D.-S. Wang, H.-F. Jiang, W. Zhang, and S.-G. Zhang, Chiral supersolid in spinorbit-coupled Bose gases with soft-core long-range interactions, Phys. Rev. Lett. 121, 030404 (2018).

[45] I. B. Spielman, Raman processes and effective gauge potentials, Phys. Rev. A 79, 063613 (20109).

[46] J. B. Balewski, A. T. Krupp, A. Gaj, S. Hofferberth, R. Löw and T. Pfau, Rydberg dressing: understanding of collective many-body effects and implications for experiments, New J. Phys. 16, 063012 (2014).

[47] I. Seydi, S. H. Abedinpour, R. E. Zillich, R. Asgari, and B. Tanatar, Rotons and Bose condensation in Rydbergdressed Bose gases, Phys. Rev. A 101, 013628 (2020).

[48] Y. Zhou, Y. Li, R. Nath, and W. Li, Quench dynamics of Rydberg-dressed bosons on two-dimensional square lat- 
tices, Phys. Rev. A 101, 013427 ( 2020).

[49] Y. Zhang, G. Chen, and C. Zhang, Tunable spin-orbit coupling and quantum phase transition in a trapped Bose-Einstein condensate, Sci. Rep. 3, 1937 (2013).

[50] K. Jiménez-García, L. J. LeBlanc, R. A. Williams, M. C. Beeler, C. Qu, M. Gong, C. Zhang, and I. B. Spielman, Tunable spin-orbit coupling via strong driving in ultracold-atom systems, Phys. Rev. Lett. 114, 125301 (2015).

[51] C.-H. Hsueh, Y.-C. Tsai, K.-S. Wu, M.-S. Chang, and W. C. Wu, Pseudospin orders in the supersolid phases in binary Rydberg-dressed Bose-Einstein condensates, Phys. Rev. A 88, 043646 (2013).

[52] F. Dalfovo, S. Giorgini, L. P. Pitaevskii, and S. Stringari, Theory of Bose-Einstein condensation in trapped gases, Rev. Mod. Phys. 71, 463 (1999).

[53] R. M. Wilson, C. Ticknor, J. L.Bohn, and E. Timmermans, Phys. Rev. A 86, 033606 (2012).

[54] M. Abad and A. Recati, A study of coherently coupled two-component Bose-Einstein condensates, Eur. Phys. J. D 67, 148 (2013).

[55] R. Wu and Z. Liang, Beliaev damping of a spin-orbitcoupled Bose-Einstein condensate, Phys. Rev. Lett. 121, 180401 (2018).

[56] Y. Li, L. P. Pitaevskii, and S. Stringari, Quantum tri- criticality and phase transitions in spin-orbit coupled Bose-Einstein condensates, Phys. Rev Lett. 108, 225301 (2012).

[57] X.-L. Chen, J. Wang, Y. Li, X.-J. Liu, and H. Hu, Quantum depletion and superfluid density of a supersolid in Raman spin-orbit-coupled Bose gases, Phys. Rev. A 98, 013614 (2018).

[58] S. Saccani, S. Moroni, and M. Boninsegni, Excitation spectrum of a supersolid, Phys. Rev Lett. 108, 175301 (2012).

[59] T. Ozawa and G. Baym, Stability of ultracold atomic Bose condensates with Rashba spin-orbit coupling against quantum and thermal fluctuations, Phys. Rev. Lett. 109, 025301 (2012).

[60] X. Cui and Q. Zhou, Enhancement of condensate depletion due to spin-orbit coupling, Phys. Rev. A 87, 031604(R) (2013).

[61] Z. Chen and Z. Liang, Ground-state phase diagram of a spin-orbit-coupled bosonic superfluid in an optical lattice, Phys. Rev. A 93, 013601 (2016).

[62] L. Liang and P. Törmä, Quantum corrections to a spin-orbit-coupled Bose-Einstein condensate, Phys. Rev. A 100, 023619 (2019).

[63] C. A. Müller and C. Gaul, Condensate deformation and quantum depletion of BoseEinstein condensates in external potentials, New J. Phys. 14, 075025 (2012) . 